\title{
Salomé et la danse avec les mots
}

Les Moralités Légendaires de Jules Laforgue

Adélaïde Jacquemard-Truc

\section{(2) OpenEdition}

Journals

Édition électronique

URL : http://journals.openedition.org/aes/382

DOI : 10.4000/aes.382

ISSN : 2258-093X

Éditeur

Laboratoire LISAA

Référence électronique

Adélaïde Jacquemard-Truc, "Salomé et la danse avec les mots », Arts et Savoirs [En ligne], 3 | 2013,

mis en ligne le 15 février 2012, consulté le 19 avril 2019. URL : http://journals.openedition.org/aes/382 ; DOI : 10.4000/aes.382

Ce document a été généré automatiquement le 19 avril 2019

Centre de recherche LISAA (Littératures SAvoirs et Arts) 


\title{
Salomé et la danse avec les mots
}

Les Moralités Légendaires de Jules Laforgue

\author{
Adélaïde Jacquemard-Truc
}

1 «Salomé » est la première adaptation comique de Laforgue. Cette nouvelle ouvre une voie qui aboutira à la publication des Moralités Légendaires, une série de réécritures comiques de mythes ou d'œuvres littéraires bien connues. Pour donner une version contemporaine et quelque peu iconoclaste de chacun des mythes qu'il reprend, Laforgue fait un choix curieux : il s'appuie sur le genre médiéval de la moralité, qu'il affiche et qu'il détourne. Les courtes pièces de théâtre à vocation édifiante deviennent sous sa plume des nouvelles dont certaines, notamment "Salomé », se terminent effectivement sur une moralité. Si le terme moralité rentre aisément en consonance avec le travail d'adaptation d'une source biblique, le lecteur est plus désarçonné lorsqu'il découvre le traitement potache réservé à l'histoire de la mort de Jean-Baptiste.

2 La surprise est d'autant plus grande que l'histoire de Jean-Baptiste et de Salomé est très fréquemment travaillée par les artistes du XIX siècle, au point de constituer une véritable vogue. Écrire une Salomé est alors un morceau de bravoure pour toute une école esthétique (littéraire, mais également picturale et musicale). La vogue des Salomé correspond à un moment charnière de l'histoire littéraire française, à la fois en crise et en quête de sources nouvelles. C'est sans doute ce qui explique que tant d'artistes se tournent vers la figure biblique de la jeune danseuse galiléenne.

3 Ce phénomène a une conséquence directe sur le texte de Laforgue: le processus d'adaptation se caractérise par sa complexité, car il joue sur deux niveaux. D'une part, il adapte les textes sacrés, et d'autre part, il se situe dans cette série d'adaptations contemporaines. Les hypotextes bibliques ne sont ici qu'une source mineure d'inspiration, alors que se lit à tout moment l'influence des nombreuses réécritures issues des mouvements symbolistes et décadents. La référence à l'« Hérodias» de Flaubert est omniprésente ; mais sans doute l'« Hérodiade » de Mallarmé et le passage que Huysmans consacre à Salomé dans A rebours jouent-ils aussi un rôle important.

4 L'originalité de Laforgue consiste à donner un ton comique à une intrigue qui s'y prête mal, du fait de sa trame et de son origine religieuse. Traité avec déférence par de nombreux artistes au $\mathrm{XIX}^{\mathrm{e}}$ siècle, le personnage de Salomé touche manifestement 
l'imaginaire de la fin-de-siècle. La danseuse incarne un type féminin très présent dans l'imaginaire décadent, celui de la femme castratrice. Elle est également une figure emblématique de la création artistique et plus précisément de la poésie, à l'époque de l'avènement de la poésie moderne. La moralité de Laforgue s'inscrit dans la continuité des œuvres de ses maitres et reprend à son compte l'esthétique d'une époque ; et pourtant, cette somme se désolidarise en partie de ses devanciers, en dénonçant par le rire les obsessions de son temps.

5 S'il existe à l'évidence des enjeux littéraires propres à la réécriture ${ }^{1}$, le cas de la «Salomé » de Laforgue pose des questions encore plus spécifiques : quels sont les motifs de la réécriture lorsque le nouveau texte s'inscrit dans une mode? On pourrait soupçonner Laforgue de profiter d'une opportunité littéraire ; mais la tonalité comique de sa nouvelle vient introduire une dissonance dans la série des Salomé de la fin de siècle. De fait, notre moralité se moque de l'époque contemporaine, bien plus qu'elle ne réécrit l'épisode biblique, qui apparaît comme un prétexte. Entre pastiche et parodie, Laforgue conduit une réflexion sur la pratique de la littérature, à la suite mais aussi à l'encontre des auteurs de la deuxième moitié du XIX siècle.

\section{Héritages et appropriation}

L'acte de réécriture constitue, dans une certaine mesure, une limitation de la créativité de l'auteur ; en choisissant de reprendre un épisode biblique, Laforgue hérite d'une intrigue à partir de laquelle il propose une variation. Mais la «Salomé» de Laforgue est plus qu'une réécriture d'un épisode biblique.

7 La source biblique de notre moralité est double : on trouve ce récit chez Marc $(6,14-29)$ et Matthieu (14, 1-12). Des hypotextes bibliques, Laforgue conserve quelques grandes structures: l'action se situe dans le palais d'un Tétrarque, «infime proconsul romain $»^{2}$, dans lequel se trouve emprisonné un prophète, appelé alternativement Iaokanann ou Jean-Baptiste - transcription du nom hébreu ou son équivalent français. Au cours d'un banquet, le Tétrarque fait à sa belle-fille l'imprudente promesse de lui donner tout ce qu'elle lui demandera, en guise de remerciement pour avoir su le divertir. Tenu par son serment, le Tétrarque doit donc livrer à la jeune fille la tête décollée du prophète. Deux élément essentiels dans les évangiles disparaissent en revanche de la moralité de Laforgue : le personnage d'Hérodias, la mère de Salomé, est totalement absent, tout comme la danse de la jeune fille. Aux intertextes évangéliques, il faut rajouter une dernière source antique : c'est Flavius Josèphe qui donne à la jeune fille le prénom de Salomé - nom qui lui est resté par la suite, consolidé par la fascination exercée par le personnage au Moyen Âge.

8 Si le récit de Laforgue hérite d'une intrigue biblique, c'est à l'« Hérodias » de Flaubert qu'il doit sa trame narrative. Daniel Grojnowski et Henri Scepi ont bien montré, dans leur introduction aux Moralités Légendaires ${ }^{3}$, à quel point Laforgue copie la construction du récit de Flaubert. Il divise « Salomé » en quatre parties, et emprunte tout le déroulement des trois premières au récit de Flaubert. Seule la dernière correspond à une invention de sa part. La moralité de Laforgue est donc très largement redevable au texte de Flaubert qui est manifestement le point de départ de son projet, comme le montre cette lettre à Charles Henry: «Tu connais l'Hérodias de Flaubert? Je viens de finir une petite Salomé de moi $»^{4}$. 
9 Allons plus loin, et notons que la quatrième partie ajoutée par Laforgue, qui narre la mort de Salomé, est très proche de la chute de Salammbô - le rapprochement des deux héroönes étant probablement facilité par l'homophonie de leurs noms ${ }^{5}$. La critique a déjà remarqué que la phrase qui conclut notre moralité est une variation de la clausule du roman de Flaubert ${ }^{6}$; mais les ressemblances ne s'arrêtent pas là. La scène finale du roman présente la princesse "penchée sur la balustrade » de la citadelle, vers laquelle s'élance, éperdu d'amour, un Mâtho écorché vif ( «Il n'avait plus, sauf les yeux, d'apparence humaine; c'était une longue forme complètement rouge ${ }^{7}$ ). Ce tableau n'est pas sans rappeler notre Salomé, «accoudée au parapet de l'observatoire " ${ }^{8}$, s'apprêtant à jeter à la mer un objet tout aussi macabre : la tête grimaçante du Baptiste.

10 Si Flaubert est la source principale de Laforgue, il reste important de situer la moralité dans son contexte littéraire, particulièrement riche. Laforgue emprunte sans doute des traits de son héroïne au poème de Mallarmé, comme la transformation de Salomé en oratrice, ainsi que la dimension métalittéraire de son discours - nous y reviendrons. L'influence mallarméenne est également sensible à des références ponctuelles : le «rêve de kermesse faunesque ", immédiatement suivi de la mention des "cygnes», qui semblent faire allusion respectivement à L'Après-midi d'un faune et à Hérodiade.

11 Laforgue, réécrivant l'histoire de Salomé, en modifie également les donnéestransgression nécessaire à la constitution de son texte comme œuvre d'art. La moralité de Laforgue n'est pas un simple exercice de style plus ou moins plagiaire, mais une œuvre authentique, capable de transformer la tradition littéraire et de la revivifier. Les transformations apportées par notre auteur sont considérables et contrebalancent son utilisation d'une trame de seconde main. Salomé devient la fille du Tétrarque et non sa belle-fille (Laforgue conserve cependant la fascination du tétrarque pour la princesse ; l'interprétation incestueuse, sans être tout à fait explicite, demeure possible $\left.{ }^{10}\right) . \mathrm{Ce}$ changement de statut apparaît comme la conséquence de la disparition d'Hérodias. Un tel choix bouleverse le sens originel des récits bibliques, en supprimant l'ambiguïté du statut de Salomé. Selon la tradition évangélique, Hérodias est la vraie responsable de la mise à mort du prophète: elle utilise sa fille pour se venger du Baptiste, qui lui reproche publiquement son mariage incestueux avec le Tétrarque. Marc acquitte implicitement Salomé, qui n'est qu'« une fillette ». Flaubert a conservé dans sa trame la machination d'Hérodias, ce qui justifie qu'elle soit, plutôt que Salomé, le personnage éponyme. Chez Laforgue, Salomé devient la seule responsable de la mort de Iaokanann ${ }^{11}$ : elle accède à la parole, et prend seule ses décisions. Il faut souligner l'importance de ce choix : avant la pièce d'Oscar Wilde, Salomé devient la femme castratrice qu'elle est restée dans l'imaginaire moderne. Ce transfert justifie que Laforgue en fasse le personnage éponyme de la nouvelle - même si son nom, qui signifie « paix » en hébreu, semble contredire son nouveau statut d'homicide.

Si Salomé devient la coupable de la mort d'Iaokanann, c'est que, comme Hérodias dans la version originelle du mythe, elle le perçoit comme une menace. Mais au différend sur l'immoralité d'un mariage succède une simple intrigue amoureuse : Salomé s'est laissée séduire par le prophète et cherche à faire disparaître celui qui pourrait la compromettre.

Ce dernier élément ouvre la porte à une lecture triviale du personnage de Salomé et de la nouvelle qui porte son nom. Le thème de l'impudicité reçoit chez Laforgue un nouveau traitement, qui indique d'ores et déjà la dominante comique de la nouvelle. À un travail d'adaptation littéraire à plusieurs niveaux de référence, la virtuosité de Laforgue ajoute une réécriture sur le ton parodique et comique. 


\section{Une parodie fin-de-siècle} ne puisse pas les prendre au sérieux. Le lecteur se trouve donc placé dans rapport complexe à ce texte à la fois poétique et ridicule. Par exemple, le narrateur emprunte quelques images à la vogue de l'écriture orientaliste, notamment lorsqu'il évoque le " kriss sacré ${ }^{12}$ du bourreau, ou les « faces non épilées ${ }^{13}$ des Princes du Nord. L'emploi du nom Iaokanann pour désigner le prophète est un autre trait de couleur locale, puisque Laforgue remonte à la racine hébraïque du nom. Mais cet orientalisme, tout juste esquissé, se limite à quelques formules sporadiques. En reprenant quelques traits saillants d'une écriture de l'orient, Laforgue en épingle les clichés et la désigne comme une écriture figée. Lui-même se situe de cette manière dans une dynamique ambivalente, entre inscription et mise à distance.

La multiplication des traits précieux dans le texte de Laforgue entraîne un effet de saturation, qui empêche de les prendre au sérieux. Longueur des phrases, rythme des propositions, antéposition des adjectifs, vocabulaire recherché : toute une panoplie d'effets est mobilisée au service de ce ton trop grandiloquent pour prétendre au grand style. Nombreux sont les paragraphes composés d'une seule phrase, et parfois Laforgue semble compliquer leur syntaxe à plaisir.

Et alors, dans cette aérienne salle jonchée de joncs jaune jonquille, entonnellée tout autour d'assourdissantes volières, un jet d'eau central fusant percer là-haut un bariolé vélarium de caoutchouc blanc sur lequel on l'entendait retomber ensuite en belle pluie frigide et claquante, ça fit, le long de tables demi-circulaires, dix rangs de lits parés chacun selon la science du convive - et, en face, une scène d'Alcazar, merveilleusement profonde, où la fleur des baladins, jongleurs, beautés et virtuoses des Iles devait venir s'effeuiller ${ }^{14}$.

En plaçant les adjectifs devant les noms dans des tournures inattendues, ou en jouant de la paronomase, Laforgue crée un effet de raffinement sur lequel la lecture s'attarde ; ainsi, les locutions "bariolé velarium» ou "joncs jaune jonquille» attirent l'attention. Le narrateur fait également appel à un lexique recherché, par exemple lorsqu'il décrit les 
《coruscants brocards lamés $»^{15}$ des atours des insulaires. Il joue également des effets d'homophonie, en accumulant les termes proches de l'homéoptotes (ésotérique, eurythmique, tétrarchique, mystique).

18 La multiplication des adverbes vient également enjoliver les descriptions. Ainsi, les multiples apparitions de Salomé donnent lieu au même commentaire; seul l'adverbe initial varie. Le narrateur s'amuse manifestement à décrire Salomé d'abord "mélodieusement emmousselinée d'arachnéenne jonquille à pois noir ${ }^{16}$, puis "hermétiquement $»^{17}$, et enfin "décidément emmousselinée ${ }^{18}$. La variation des adverbes donne lieu à un comique de répétition - tout littéraire, puisque c'est une tournure qui se répète, et non une situation -, doublé d'une allusion grivoise. En effet, lorsqu'elle fuit la première fois, Salomé «mélodieusement emmousselinée » a été dérangée pendant son bain, interruption qui donne lieu à des «salamalecs galants $»^{19}$. Il est donc surprenant qu'on la retrouve « hermétiquement emmousselinée » alors qu'elle a à peine eu le temps de fuir. De plus, l'expression relève de l'oxymore, puisque la mousseline est par définition un tissu vaporeux. Cette contradiction dans les termes dément l'insistance du narrateur sur la pudeur du personnage. La répétition attire l'attention du lecteur sur le corps de Salomé, qui se voile et se dévoile tout à la fois, et remplace peut-être sa danse, absente de la nouvelle ${ }^{20}$. Le dernier adverbe, " décidément ", qui sert d'abord à souligner le comique de répétition, semble indiquer également la déception des intrus, rendue en focalisation interne par le narrateur ${ }^{21}$.

D'autres adverbes, qui relèvent du néologisme, viennent enrichir le texte. Le but est autant de créer un rapport libre et ludique au langage, que d'alourdir sciemment la narration, pour lui donner une tournure risible. Ainsi, «sacerdotalement $»^{22}$,

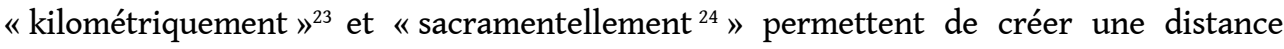
propice au comique entre le lecteur et sa lecture. Le néologisme "sacramentellement " est un exemple particulièrement parlant du processus de réécriture de Laforgue, qui allie dans une construction ludique le sacré (religieux ou littéraire) et le populaire. Jouant avec les sonorités de la langue, le narrateur forge volontiers d'autres types néologismes pour enrichir le récit: ainsi, comme nous l'avons vu, Salomé n'est pas vêtue d'une robe de mousseline, mais « emmousselinée »

À travers ces choix stylistiques, Laforgue écrit contre de nombreux auteurs du dixneuvième siècle, et notamment Flaubert, dont il parodie manifestement le style - même s'il s'agit d'un certain Flaubert. Certaines références explicites ne laissent pas de doute : Laforgue tourne en dérision les titres que Flaubert donne à ses personnages. Il crée à son tour des expressions composées, mais il les multiplie de manière à surcharger le texte et leur donne, de plus, des connotations comiques. Ainsi, «l'Annonciateur des Lunes ${ }^{25}$ ou le «Chef-des-odeurs-suaves ${ }^{26}$ deviennent «l'Ordonnateur-des-mille-riens » ${ }^{27}$, «le Répétiteur des Gynécées et Sélections ${ }^{28}$, ou encore le "Conservateur des Symboles $»^{29}$. Notre moralité se situe en un lieu mal défini entre la parodie et le pastiche. Elle tient à la fois de l'exercice de style, car il s'agit bien d'imiter le style d'un maître pour se mesurer à lui (ce qui donne un rôle valorisant à l'hypotexte), et de caricaturer ses principaux effets stylistiques, caricature qui aboutit à le tourner en dérision.

21 Le but de Laforgue est-il ici véritablement de faire rire? Le narrateur établit une connivence malicieuse, mais ces éléments ne visent sans doute pas à déclencher une franche hilarité. Le lecteur, qui est supposé disposer de la culture nécessaire pour décrypter ce travail de réécriture et de mise à distance, s'amuse sans doute de l'impertinence du disciple envers les maîtres; mais il ne s'agit pour l'instant que d'un 
sourire intellectuel. De tels effets de langage relèvent de l'humour plus que du comique : l'usage inapproprié du style noble désamorce l'ancienne solennité et fait sourire. Comme dans l'Affaire Lemoine de Proust, l'écriture comique est une arme en vue d'une critique critique d'une excessive confiance dans le pouvoir de la littérature, sans doute.

\section{Un comique des contrastes}

22 Le comique de "Salomé » ne repose pas exclusivement sur le détournement d'un style noble ; Laforgue utilise également, à la manière d'un contrepoint, un registre bas : sa réécriture comique de l'épisode biblique se fonde essentiellement sur une esthétique des contrastes, et bien souvent, le registre noble emprunté aux auteurs contemporains subit une dégradation qui tire le texte vers un comique plus léger.

Le premier trait de cette dégradation est le ton adopté par le narrateur, qui rappelle celui des bonimenteurs de foire. La présence d'une subjectivité dans la narration est marquée d'emblée par l'utilisation de nombreuses exclamatives: ainsi, dès le troisième paragraphe, la description du palais se transforme en invocations à caractère descriptif ce qui suppose la présence d'un locuteur affecté par la vision du palais : «Titanique masse funèbre veinée de blême $! »^{30}$, ou plus loin " Oh, le Tétrarque sur la terrasse, cariatide des dynasties! $»^{31}$. L'usage d'un style ampoulé apparait d'autant plus inapproprié qu'il est placé dans la bouche d'un narrateur qui se présente comme un badaud naïf et fasciné par le spectacle du palais. De même, la première véritable apparition de Salomé donne lieu à des exclamations : «Oh, le petit Messie à matrice! Que sa tête lui était onéreuse ! $^{32}$.

À l'affirmation de la voix du narrateur correspond une intégration du lecteur dans la fiction. La situation d'énonciation qu'il crée englobe implicitement le lecteur, par l'emploi de la deuxième personne du pluriel, dans des formules comme: «les volières [...] se turent à regret quand la musique commença à vous accompagner le repas $»^{33}$, ou « la voix dure de Salomé vous le redressa vivement $»^{34}$. L'ajout de ces pronoms explétifs donne un ton oral et populaire au propos. De même, l'effet d'immédiateté est entretenu par l'emploi des infinitifs de narration, qui dynamisent le récit en lui donnant une tournure souple, proche de la parole orale: "Et de circuler alors en plein ciel, par de menues phrases d'admiration suffoquée $»^{35}$. Enfin, le récit devient discours, lorsque le narrateur décrit l'installation de Salomé sur la scène de l'Alcazar, et commente : "Elle va peut-être raconter des choses, après tout? $»^{36}$. Le statut de narrateur omniscient disparaît alors : tout comme le lecteur, le narrateur attend de découvrir la suite de l'action. Il devient un spectateur comme les autres, ce qui renforce l'impression d'un récit conduit par un simple badaud.

Ce statut original du narrateur permet à Laforgue d'introduire des éléments comiques qui eussent été inimaginables sinon. Il use de multiples procédés pour ramener le ton de la moralité vers un registre bas. Le narrateur joue avec les sons autant qu'avec les mots ; ainsi nous décrit-il la salle du spectacle " jonché[e] de joncs jaune jonquille » ${ }^{37}$, en forçant l'allitération jusqu'à la paronomase. Les jeux de mots sont l'occasion de ménager des glissements de sens et de créer la surprise. Ainsi, les crabes de l'Aquarium «s'empêtrent en couples avec de petits yeux rigoleurs de pince-sans-rire $»^{38}$. Le soleil est quant à lui rebaptisé «Lampyre de l'Empyrée $»^{39}$, titre majestueux fondé sur l'homophonie presque parfaite des deux termes employés. Mais sous la pompe apparente se cache une dégradation toute burlesque : mot-à-mot, l'expression signifie «vers luisant des plus 
hautes sphères ». Ces exemples forts différents révèlent un même rapport ludique au langage, qui lui aussi favorise le rire.

Une autre ressource importante du comique dans «Salomé » est le mélange des registres, qui s'opère à nouveau dans le sens de la dégradation. Ainsi, il n'est pas sans saveur de lire : «Le Tétrarque biberonnait son houka de midi » $^{40}$. L'arrêt de mort de Iaokanann est prononcé de manière on ne peut plus triviale : sa tête sera "Adjugée ! » ${ }^{41}$ à l'encan. Le narrateur manifeste un net refus des effets poétiques trop attendus, notamment lorsqu'il écrit : «Et devant lui, la mer, la mer, toujours nouvelle et respectable, la Mer puisqu'il n'y a pas d'autre nom pour la nommer $\aleph^{42}$. Le narrateur semble ici déplorer l'absence de terme poétique propre à ennoblir la réalité décrite - faute de quoi, il la gratifie d'une majuscule. Cette épanalepse est sans doute une référence implicite à l'Azur mallarméen. Il souligne de ce fait l'incontournable trivialité du monde et du langage, sur laquelle achoppe le travail littéraire. Contre une tradition poétique qui cherche à enjoliver son objet, le narrateur démasque la vacuité du langage poétique. Mais à l'opposé du ton énergique employé par Hugo ${ }^{43}$, Laforgue choisit la dérision.

Le troisième procédé comique employé par Laforgue est le déplacement; il fait rire son lecteur en donnant à l'épisode biblique un cadre inattendu, fort différent de la Palestine du début de l'ère chrétienne. La scène se passe en juillet, un jour de « fête nationale " ${ }^{44}$, allusion à peine voilée aux célébrations de la prise de la Bastille. Le narrateur fait référence à Napoléon ${ }^{\text {er455. }}$. Les Princes du Nord devisent quant à eux de "l'autorité armée $»^{46}$ (nous sommes en pleine crise du boulangisme), de la «religion» et de la « concurrence internationale $»^{47}$. Mais l'on se rend compte que le déplacement n'est pas seulement spatio-temporel: Laforgue transpose l'univers biblique dans le monde du théâtre. Il y a bien sûr la troisième partie, située dans une salle de spectacle et sur la scène de l'Alcazar. Mais le château du Tétrarque évoque lui-même un décor de théâtre, car il a sa face cachée. Salomé s'enfuit une première fois en se laissant « glisser, par un jeu de poulies, dans le vide, vers d'autres étages ${ }^{48}$ : cet équipement rappelle les moyens techniques mis en œuvre au théâtre. Le narrateur nous décrit également le phare du palais comme un "phare d'opéra-comique $»^{49}$. Le palais est donc lui aussi un monde factice, destiné à donner l'illusion du vrai.

Ces procédés comiques, au-delà de leurs effets propres, ont une seconde vertu: par contraste, ils rendent plus sensible la tonalité comique de la moralité, et clarifient son statut de parodie-pastiche. Il est impossible, dans ce contexte, de prendre au sérieux le recours au style noble.

\section{Mise en crise du langage littéraire}

29 Le choix que fait Laforgue de remplacer la danse de Salomé, élément crucial de l'épisode biblique, par un monologue déplace l'intérêt de l'intrigue. Le trait fondamental de la moralité devient la mise en abyme : le rapport au langage est mis en fiction, mis en mots.

Chez Laforgue, la danse est représentée par d'autres personnages que Salomé. Le motif n'est donc pas écarté, mais volontairement déplacé, alors que le personnage principal gagne un nouvel attribut : elle possède une lyre - autre élément emprunté à Flaubert dont elle s'accompagne pendant son vocéro (la critique a rebaptisé ce passage en empruntant le terme qui désigne un chant funèbre corse). La récitante casse son instrument une fois le numéro fini : on peut lire dans ce geste une référence plus précise 
au lyrisme, dont l'impossibilité serait signalée. Salomé devient donc une figure du poète, et sans doute plus précisément du poète lyrique. Cette piste est d'autant plus signifiante que d'autres indices viennent la conforter : la tête décollée d'Iaokanann est comparée à celle d'Orphée ${ }^{50}$. De même, dès la première partie de la nouvelle, apparaît l'image du " soleil Aède ${ }^{51}$ Pourtant, notre poétesse pose problème. Le symbole de la lyre entre en contradiction avec le morceau déclamée par l'héroïne, que le narrateur qualifie de "garulement $~_{52}$ - le morceau évoque donc le cri du geai. Laforgue construit donc une représentation complexe du poète; tous ces indices nous mettent sur la piste d'une lecture métatextuelle de la nouvelle.

31 La réflexion sur la nature de l'art sous-tend toute la nouvelle. Cette réflexion permet de mieux comprendre le rapport complexe que Laforgue entretient avec l'écriture qu'il parodie : s'il raille les excès d'ornements dans le style de Flaubert et des auteurs du XIX ${ }^{\mathrm{e}}$ siècle, il y voit aussi un signe d'excellence artistique. On trouve la trace de cette ambivalence dans le texte, lorsque les Princes du nord hésitent à offrir au Tétrarque leur collier de la toison d'or : "La nullité artistique de ce collier, sautait, surtout ici, aux yeux » ${ }^{53}$. Le raffinement, poussé à l'extrême, est une réalité ambiguë, qui fascine même si elle confine au ridicule. Serait-il excessif de voir dans le Tétrarque une image de Flaubert dans son palais d'orientalisme (Salammbô, «Hérodias»), visité par un barbare curieux venu d'un pays bien moins raffiné, Laforgue, hésitant à lui remettre le piètre hommage de sa toison d'or?

Le «vocéro » de Salomé est sans doute la clef qui détermine la lecture de la moralité. En effet, la réécriture de Laforgue est construite autour d'une déception: le lecteur attend le passage de la danse de Salomé, escamoté au profit de ce que le narrateur nomme un «garulement mystique ». La déception est d'autant plus grande que le propos de Salomé s'avère incompréhensible, au moins à première lecture - alors même que Salomé parle, dans une expression provocatrice, du Néant « limpide comme tout $»^{54}$. Ici aussi, Laforgue semble viser une tendance de son époque, qui a donné lieu à des controverses entre écrivains: l'opacité. Les auteurs parodiés sont ici les poètes de la fin-de-siècle, essentiellement des symbolistes; on pense notamment à Mallarmé, auquel le texte fait allusion. La Salomé « hermétiquement emmousselinée » de notre moralité est peut-être une image métaphorique de la poésie dite, précisément, hermétique. Certains thèmes de prédilections de ces auteurs sont repris et résumés dans des adjectifs repris mal à propos, comme dans les expressions «les Iles Blanches ésotériques $»^{55}$, «tirelire mystique $»^{56}$. Mais, malgré cette parodie, Laforgue s'inscrit dans la lignée du symbolisme en faisant de sa moralité le lieu d'une recherche sur ce qu'est l'inconscient. Ainsi les personnages incarnés par les clowns s'appellent «l'Idée, la Volonté, l'Inconscient ${ }^{57}$, selon les concepts philosophiques chers à Hartmann et Schopenhauer. Le vocéro s'en prend aux «sectaires de la conscience $»^{58}$, et affirme que «tout s'étire hors du Moi ${ }^{59}$. Il ne peut qu'être obscur, parce que le langage clair de la raison est par nature incompatible avec l'exploration des formes spécifiques de l'inconscient. La seule différence avec les déclarations sur l'inconscient d'un Maeterlinck consiste dans le refus obstiné du sérieux, rappelé par un petit « rire toussotant » de Salomé, « pour faire assavoir que surtout fallait pas croire qu'elle se prenait au sérieux $»^{60}$. Salomé est ici manifestement une image de Laforgue, qui joue des contradictions de son temps sans prétendre les dépasser.

33 Le langage, dans cette moralité, est un véritable objet de désir, comme le montre la réaction du Tétrarque. L'enjeu de la réécriture de "Salomé » est de conquérir une légitimité littéraire et de s'égaler à d'imposants modèles. Et pourtant, là où on attendrait 
un morceau de bravoure, Salomé ne prononce qu'un «garulement». Pour Laforgue, écriture comique et déception semblent aller de pair. Mettant au centre de sa nouvelle un discours obscur et sans fondements, notre auteur semble renoncer définitivement aux sommets de la littérature, dont il ne peut proposer qu'une version dérisoire. Ainsi, la disparition de la danse de Salomé peut s'interpréter comme un aveu d'échec. Le monde littéraire semble se refermer sur lui-même : ne parvenant plus à dépasser ses propres contours, il perd prise avec le réel. Décrire une chorégraphie au moyen de mots est assurément une gageure; Laforgue esquive cette difficulté en se rabattant sur de la parodie. Le comique permet à Laforgue de continuer une tradition littéraire en désamorçant toute rivalité avec ses prédécesseurs - puisque le texte comique affirme d'emblée qu'il ne se prend pas au sérieux.

Sans doute cette Salomé poétesse est-elle également l'emblème d'une époque où la littérature se cherche. Soulignons d'ailleurs qu'avant Laforgue, Mallarmé avait déjà fait d'Hérodiade au miroir le symbole de la réflexivité de l'écriture littéraire. Ainsi, on peut comprendre la réaction du Tétrarque face au vocéro comme une nouvelle mise-en-abyme : celle des contemporains de Laforgue face à une littérature bavarde et complaisante. Pour Laforgue, « Hérodias » apparait comme un point de non-retour : c'est un sommet que l'on ne peut dépasser (ni même reproduire) sans verser dans le ridicule. L'adaptation comique de "Salomé » traduit chez notre auteur une véritable « crise de prose », comparable au tournant que Mallarmé diagnostique dans la poésie post-hugolienne.

«Salomé » est donc un texte qui présente les difficultés de l'entrée dans une nouvelle ère littéraire, qui se caractérise comme un "après", marquée par d'imposants chefsd'œuvre. Dans cette moralité, Laforgue mène une réflexion sur le langage, ce qui fait de lui un continuateur de Mallarmé et de son Hérodiade. Ce texte apparaît comme une critique de la littérature de son temps, mais est aussi une critique de soi-même; et en ce sens, son statut est problématique. Le lecteur aboutit à une sorte d'impasse : si l'on ne peut plus se permettre le beau style, comme le laisse entendre notre auteur par sa parodie, la littérature ne peut que déchoir. Les écritures du passé sont devenues risibles, et on ne voit pas, dans cette moralité, par quels moyens nouveaux la littérature pourrait se développer. La critique de Laforgue ne débouche sur rien, sinon sur un moment de réflexion ludique. Mais écrire en montrant les limites de la littérature, c'est encore écrire et c'est encore de la littérature. "Salomé " est une méditation sur l'impuissance de l'écriture, dans laquelle le comique semble avoir pour rôle de redonner sens à une œuvre en quête de légitimité, de redonner forme à une impossibilité d'écrire. La parodie sert de transition. Charnière entre deux siècles, cette écriture comique est sans doute vécue comme un espace gagné sur le silence - dans l'attente d'un renouveau plus profond.

\section{NOTES}

1. Cf. G. Genette, Palimpseste, Seuil, Paris, 1982, p. 446 sq.

2. J. Laforgue, Moralités Légendaires, présentation par Daniel Grojnowski et Henri Scepi, GarnierFlammarion, Paris, 2000, p. 133. 
3. Ibid., p. 25-26.

4. J. Laforgue, Euvres Complètes., t. II, L’Âge d'homme, Lausanne, 1986, p. 767.

5. Mireille Dottin-Orsini montre, dans « Laforgue fumiste : Salomé Floupette », que Laforgue joue, dans ses Fleurs de bonne volonté («Dimanches»), sur cette proximité pour confondre les deux héroïnes. Raison, dérision, Laforgue, Romantisme, 1989, n 64., p. 20.

6. Voir M. Dottin-Orsini., « Laforgue fumiste : Salomé Floupette », ibid., p. 21.

7. G. Flaubert, Salammbô, Folio classiques, Paris, 1970, p. 467.

8. J. Laforgue, Moralités Légendaires, op. cit., p. 151.

9. Ibid, p. 139.

10. «Puis il souriait à tous, en père heureux, l'air de dire : vous allez voir ce que vous allez voir ", mettant les princes ses hôtes au courant, de façon fort décousue, où ceux-ci comprirent que, pour faire un sort à la petite personne en question, la Lune s'était saignée aux quatre veines, et qu'on la tenait d'ailleurs généralement (il y avait eu une Concile là-dessus) pour la sœur de lait de la Voie Lactée (tout pour elle !). » Ibid., p. 147. Voir également : « Oh! Continue, continue, dis tout ce que tu sais ! geignait Emeraude-Archetypas, battant des mains comme un enfant ». Ibid., p. 149.

11. Encore qu'elle n'a guère de mal à convaincre son père de lui donner satisfaction.

12. J. Laforgue, Moralités Légendaires, op. cit., p. 135.

13. Ibid.

14. Ibid., p. 144.

15. Ibid., p. 138.

16. Ibid., p. 138.

17. Ibid., p. 139. La même phrase se retrouve p. 146.

18. Ibid., p. 143.

19. Ibid., p. 138.

20. Sans faire de ce passage la source nécessaire de «la danse de sept voiles », il est frappant de remarquer que le Laforgue et Wilde associent tous les deux Salomé au motif du voile.

21. Ce regard curieux est ensuite relayé par le narrateur lui-même, qui décrit une mousseline «qui, s'agrafant çà et là de fibules diverses, laissant les bras à leur angélique nudité, formait entre les deux soupçons de seins aux amandes piquées d'un œillet, une écharpe brodée de ses dix-huit ans ». Ibid., p. 146-147.

22. Ibid., p. 138.

23. Ibid., p. 139.

24. Ibid, p. 144.

25. G. Flaubert, op. cit., p. 185.

26. J. Laforgue, Moralités Légendaires, op. cit., p. 225.

27. Ibid., p. 137.

28. Ibid.

29. Ibid.

30. Ibid., p. 133.

31. Ibid., p. 134.

32. Ibid., p. 147.

33. Ibid., p. 144.

34. Ibid., p. 150.

35. Ibid., p. 138.

36. Ibid., p. 147.

37. Ibid., p. 144.

38. Ibid., p. 140.

39. Ibid., p. 134.

40. Ibid., p. 135.

41. Ibid., p. 151. 
42. Ibid., p. 134.

43. V. Hugo, Les Contemplations, Garnier, Paris, 1969, Livre I, « Réponse à un acte d'accusation », p. 23 : « J'ai dit à la narine : eh mais ! tu n'es qu'un nez ».

44. J. Laforgue, Moralités Légendaires, op. cit., p. 135.

45. Ibid., p. 142.

46. Ibid., p. 145.

47. Ibid., p. 145-146.

48. Ibid., p. 138.

49. Ibid., p. 133.

50. Ibid., p. 152 : « la tête de Jean (comme jadis celle d'Orphée) ».

51. Ibid., p. 134.

52. Ibid., p. 149.

53. Ibid., p. 144.

54. Ibid., p. 148.

55. Ibid., p. 133.

56. Ibid., p. 136.

57. Ibid., p. 145.

58. Ibid., p. 150.

59. Ibid.

60. Ibid., p. 147-148.

INDEX

Mots-clés : comique, Laforgue (Jules), réécriture, parodie

\section{AUTEUR}

\section{ADÉLAIIDE JACQUEMARD-TRUC}

Université Paris-Est Marne-la-Vallée 